\title{
Bogomol'nyi-Prasad-Sommerfield solitons in M5-brane worldvolume theory with a constant three-form field
}

\author{
Donam Youm* \\ Theory Division, CERN, CH-1211, Geneva 23, Switzerland \\ (Received 13 September 2000; published 16 January 2001)
}

\begin{abstract}
We study Bogomol'nyi-Prasad-Sommerfield solutions for a self-dual string and a neutral string in M5-brane worldvolume theory with a constant three-form field. We further generalize such solitons to superpose with a calibrated surface. We also study a traveling wave on a calibrated surface in the constant three-form field background.
\end{abstract}

DOI: 10.1103/PhysRevD.63.045004

PACS number(s): 11.27.+d, 04.20.Jb, 11.15.Tk, 11.30.Pb

\section{INTRODUCTION}

Recently, noncommutative theories have received renewed interest, after it was found that noncommutative theories can be naturally realized in $\mathrm{M}$ theory compactified in the presence of constant three-form field background [1] and within string theories as the worldvolume theory of a D-brane with a nonzero constant Neveu-Schwarz (NS) $B$ field $[2,3]$. In the case of a D-brane with a constant magnetic NS $B$ field, one can take a decoupling limit of the D-brane worldvolume theory to achieve a noncommutative field theory with space-space noncommutativity. However, in the case of an electric NS $B$-field background, one cannot take a zero slope limit in such a way that the noncommutativity parameter is nonzero in order to obtain a field theory with space-time noncommutativity since the electric NS $B$ field cannot be scaled to infinity due to its critical value, beyond which the open string parameters are not well defined [4]. It is conjectured $[4,5]$ that nearby a critical electric $B$ field with an appropriate scaling limit, a novel noncritical open string theory, called noncommutative open string (NCOS) theory, which decouples from closed strings (and therefore, also gravity) in the bulk, emerges. It is conjectured $[6,7]$ that the strong coupling limit of the NCOS theory in $4+1$ dimensions is the so-called open M-brane (OM) theory, which is the decoupled (from gravity) theory of light open M2-brane ending on M5-brane in the constant three-form field background.

It would, therefore, be interesting to study worldvolume solitons of the M5-brane with constant three-form field. The solitons of the brane worldvolume theories are interpreted as the intersections of the interesting branes [8], with the intersection being the source for the charge carried by the worldvolume soliton [9-11]. For example, the M5-brane worldvolume soliton counterpart to the open M2-brane ending on M5-brane is the self-dual string [12], and the three-brane soliton [13] on the M5-brane worldvolume is interpreted as the three-brane intersection of two intersecting M5-branes. The solitons in the D-brane worldvolume theory, i.e., BI solitons, or bions $[14,15,12]$, with a constant NS $B$ field, were previously studied, for example, in Refs. [16-21]. In the case

*Email address: donam.youm@cern.ch of the zero-brane soliton, corresponding to a fundamental or $\mathrm{D}$ string ending on a D-brane, force on the endpoint due to a nonzero constant NS $B$ field is shown [16] to cause the string to tilt. The nonlocality of the suspended string due to such tilting is in accordance with the fact that the D-brane worldvolume is noncommutative, which leads to uncertainty in measurement.

In this paper, we study the Bogomol'nyi-PrasadSommerfield (BPS) self-dual string and neutral (or instanton) string solitons in the M5-brane worldvolume theory with a constant three-form field, as well as their generalization on calibrated surfaces. We also show that traveling wave on the calibrated surface with a constant three-form field preserves supersymmetry. In studying such worldvolume solitons, we follow the covariant equations of motion approach [22-24] of M5-brane theory.

\section{ASPECTS OF M5-BRANE WORLDVOLUME THEORY}

We discuss the relevant aspect of the M5-brane worldvolume theory [22-27] for the purpose of fixing and defining notation. The convention for indices is as follows: The indices for the target spaces are underlined. The indices from the beginning [middle] of the alphabet refer to the tangent space [coordinate space] indices. The Latin [Greek] indices are for the bosonic [fermionic] coordinates. The primed indices are for directions normal to the M5-brane worldvolume. So, the coordinates of the target and the worldvolume superspaces are, respectively, $Z^{M}=\left(X^{m}, \Theta^{\mu}\right)$ and $z^{M}=\left(x^{m}, \theta^{\mu}\right)$, and, for example, the target space index $a[\alpha]$ is decomposed as $a$ $=\left(a, a^{\prime}\right), \quad$ with $a=0,1, \ldots, 5^{-}$and $a^{\prime}=1^{\prime}, \ldots, 5^{\prime} \quad[\bar{\alpha}$ $=\left(\alpha, \alpha^{\prime}\right)$, with $\alpha=1, \ldots, 16$ and $\left.\alpha^{\prime}=1^{\prime}, \ldots, 16^{\prime}\right]$. The fermionic indices $\alpha$ and $\alpha^{\prime}$, running from 1 to 16 , are alternatively written, respectively, as $\alpha i$ and ${ }_{i}^{\alpha}$, when appearing as subscript, and as $\alpha i$ and ${ }_{\alpha}^{i}$, when appearing as superscript, with $\alpha$ and $i$ running from 1 to 4 . For example, $\Theta^{\alpha^{\prime}}$ $\rightarrow \Theta_{\alpha}^{i}, \Theta^{\alpha} \rightarrow \Theta^{\alpha i}$, and $u_{\alpha}^{\beta^{\prime}} \rightarrow u_{\alpha i \beta}^{j}$. We denote the vielbeins of the target and the M5-brane worldvolume superspaces as $E_{M}^{A}$ and $E_{M}^{A}$, respectively. We denote the embedding matrix $\widetilde{E}_{\bar{A}}^{\bar{A}}=E_{A}^{M} \partial_{M} Z^{\underline{M}} E_{\bar{M}}^{A}$ with a tilde to avoid confusion with vielbeins. The worldvolume theory of the M5-brane is described by the $(2,0)$ tensor multiplet containing five scalars $X^{a^{\prime}}$, sixteen fermions $\Theta^{\alpha^{\prime}}$, and a self-dual field strength $h_{a b c}$. Here, 
$X^{m^{\prime}}$ and $\Theta^{\alpha^{\prime}}$ are identified as the transverse components of the target superspace coordinates $\left(X_{-}^{m}, \Theta^{\mu}\right)$.

In this paper, we consider an M5-brane embedded in flat eleven-dimensional target space with the metric $\hat{g}_{m n}=\eta_{m n}$. Just as in the case of D-brane with the NS $B$ field, the induced metric $g_{m n}=\eta_{m n} \partial_{m} X_{-}^{m} \partial_{n} X_{-}^{n}$ on the M5-brane worldvolume does not correspond to the metric on the M5-brane felt by the open M2-brane in the presence of the background three-form field strength $\mathcal{H}_{m n p}$. Here, the gauge invariant field strength $\mathcal{H}_{m n p}$ of the M5-brane worldvolume two-form potential $b_{m n}$ is given by

$$
\mathcal{H}_{m n p}=\partial_{[m} b_{n p]}+\partial_{m} X_{-}^{m} \partial_{n} X_{-}^{n} \partial_{p} X_{-}^{p} C_{\underline{m n p}},
$$

where $C_{\underline{m n p}}$ is the three-form potential in the elevendimensional supergravity, and is related to the self-dual field strength $h_{a b c}$ as

$$
\mathcal{H}_{m n p}=E_{m}^{a} E_{n}^{b} E_{p}^{c} m_{b}^{d} m_{c}^{e} h_{a d e}=e_{m}^{a} e_{n}^{b} e_{p}^{c}\left(m^{-1}\right){ }_{c}^{e} h_{a b e} .
$$

The self-duality condition on $h_{a b c}, h_{a b c}=\frac{1}{6} \epsilon_{a b c d e f} h^{d e f}$, is translated to the following nonlinear self-duality condition on $\mathcal{H}_{\text {mnp }}$ :

$$
\frac{\sqrt{-\operatorname{det} g}}{6} \epsilon_{\text {mnpqrs }} \mathcal{H}^{q r s}=\frac{1+K}{2}\left(G^{-1}\right)_{m}^{r} \mathcal{H}_{n p r},
$$

where $K \equiv \sqrt{1+\frac{1}{24} \mathcal{H}_{m n p} g^{m q} g^{n r} g^{p s} \mathcal{H}_{q r s}}$. It is suggested in Ref. [28] that it is the metric $G_{m n}=E_{m}^{a} E_{n}^{b} \eta_{a b}$ associated with $E_{m}^{a}$ that is felt by an open M2-brane ending on the M5-brane. The vielbein $e_{m}^{a}$ associated with $g_{m n}$ is related to $E_{m}^{a}$ as $E_{m}^{a}=e_{m}^{b}\left(m^{-1}\right)_{b}^{a}$, where $m_{a}^{b} \equiv \delta_{a}^{b}-2 h_{a c d} h^{b c d}$. So, the open M2-brane metric $G_{m n}$ is expressed in terms of $g_{m n}$ and $\mathcal{H}_{\text {mnp }}$ as

$$
G_{m n}=\frac{1+K}{2 K}\left(g_{m n}+\frac{1}{4} \mathcal{H}_{m p q} g^{p r} g^{q s} \mathcal{H}_{n r s}\right) .
$$

As expected, the open M2-brane metric $G_{m n}$ becomes $g_{m n}$ when $\mathcal{H}_{m n p}=0$, and reduces to the open string metric [3] on a D-brane upon double dimensional reduction.

The equations of motion of the M5-brane can be obtained by analyzing the torsion equation subject with the superembedding condition $\widetilde{E}_{\bar{\alpha}}^{a}=0$. In a flat target superspace background in the static gauge, where the fermionic field $\Theta_{-}^{\alpha}$ $=\left(\Theta^{\alpha}, \Theta^{\alpha^{\prime}}\right)$ is zero and $X^{m}=x^{m}$, the bosonic equations of motion are

$$
\begin{aligned}
G^{m n} \nabla_{m} \nabla_{n} X^{a^{\prime}} & =0, \\
G^{m n} \nabla_{m} \mathcal{H}_{n p q} & =0,
\end{aligned}
$$

where the connection in the covariant derivative $\nabla_{m}$ is defined from $g_{m n}$, i.e., $\Gamma_{m n}^{p}=\partial_{m} \partial_{n} X^{a^{\prime}} \partial_{q} X^{b^{\prime}} g^{p q} \delta_{a^{\prime} b^{\prime}}$.

We are particularly interested in solutions that preserve part of supersymmetry. The requirement that the static gauge condition $\Theta^{\alpha}=0$ be preserved under the combined rigid supersymmetry transformation of a flat target superspace and super-reparametrization of the worldvolume induced on the target superspace leads to the following form of the supersymmetry transformation for the fermions:

$$
\delta \Theta^{\alpha^{\prime}}=-\epsilon^{\alpha}\left(\widetilde{E}^{-1}\right){ }_{\alpha}^{\beta} \widetilde{E}_{\beta}^{\alpha^{\prime}},
$$

where the nonlinearly realized symmetry parametrized by $\epsilon^{\alpha^{\prime}}$ is set to zero. Making use of the projection operators $\left(\widetilde{E}^{-1}\right)_{\underline{\alpha}}^{\beta} \widetilde{E} \frac{\gamma}{\beta}=\frac{1}{2}(1+\Gamma) \frac{\gamma}{\alpha}$ and $\left(\widetilde{E}^{-1}\right)_{\underline{\alpha}}^{\beta^{\prime}} \widetilde{E}_{\beta^{\prime}}^{\gamma}=\frac{1}{2}(1-\Gamma) \frac{\gamma}{\alpha}$, one can put the variation, $\overline{\mathrm{E}} \mathrm{q}$. (6), for $\Theta^{-\bar{\alpha}^{\prime}}$ into the form

$$
\hat{\delta} \Theta^{\alpha^{\prime}} \equiv \delta \Theta^{\gamma^{\prime}}\left(\frac{1-\Gamma}{2}\right)_{\gamma^{\prime}}^{\alpha^{\prime}}=-\frac{1}{2} \epsilon^{\gamma} \Gamma_{\gamma}^{\alpha^{\prime}} .
$$

This has to be set to zero to make the condition $\Theta^{\alpha^{\prime}}=0$ invariant under the supersymmetry transformation. In terms of the bosonic fields of the M5-brane worldvolume theory, the supersymmetry variation, Eq. (7), is expressed as

$$
\begin{aligned}
& \hat{\delta} \Theta_{\beta}^{j}=-\frac{1}{2} \epsilon^{\alpha i}\left[\operatorname{det}\left(e^{-1}\right) \partial_{m} X^{a^{\prime}}\left(\gamma^{m}\right)_{\alpha \beta}\left(\gamma_{a^{\prime}}\right)_{i}^{j}\right. \\
& -\frac{1}{3 !} \operatorname{det}\left(e^{-1}\right) \partial_{m_{1}} X^{a_{1}^{\prime}} \partial_{m_{2}} X^{a_{2}^{\prime}} \partial_{m_{3}} X^{a_{3}^{\prime}}\left(\gamma^{m_{1} m_{2} m_{3}}\right)_{\alpha \beta} \\
& \times\left(\gamma_{a_{1}^{\prime} a_{2}^{\prime} a_{3}^{\prime}}\right)_{i}^{j}+\frac{1}{5 !} \operatorname{det}\left(e^{-1}\right) \partial_{m_{1}} X^{a_{1}^{\prime}} \ldots \partial_{m_{5}} \\
& \times X^{a_{5}^{\prime}}\left(\gamma^{m_{1} \ldots m_{5}}\right)_{\alpha \beta}\left(\gamma_{a_{1}^{\prime} \ldots a_{5}^{\prime}}\right)_{i}^{j} \\
& -h^{m_{1} m_{2} m_{3}} \partial_{m_{2}} X^{a_{2}^{\prime}} \partial_{m_{3}} X^{a_{3}^{\prime}}\left(\gamma_{m_{1}}\right)_{\alpha \beta}\left(\gamma_{a_{2}^{\prime} a_{3}^{\prime}}\right)_{i}^{j} \\
& \left.-\frac{1}{3} h^{m_{1} m_{2} m_{3}}\left(\gamma_{m_{1} m_{2} m_{3}}\right)_{\alpha \beta} \delta_{i}^{j}\right] \text {, }
\end{aligned}
$$

where $\gamma_{m}=\delta_{m}^{a} \gamma_{a}$.

It is the purpose of this paper to study solitons in the M5-brane worldvolume theory in the constant three-form field $\mathcal{H}$ background. When $\mathcal{H}$ is constant up to a Lorentz transformation, the nonzero components of $\mathcal{H}$ satisfying the nonlinear self-duality condition, Eq. (3), are $\mathcal{H}_{012}$ and $\mathcal{H}_{345}$ $[3,28]$. Equivalently, nonzero components of the worldvolume field $h_{a b c}$ are

$$
h_{012}=-h_{345}=h=\text { const } \text {, }
$$

which corresponds to $\mathcal{H}_{012}=\left(h /\left(1+4 h^{2}\right)\right) \equiv \frac{1}{4} \sin \theta$ and $\mathcal{H}_{345}$ $=-\left(h /\left(1-4 h^{2}\right)\right)=-\frac{1}{4} \tan \theta$, according to Eq. (2). In the infinite momentum frame (boosted along the $x^{5}$ direction), the nonzero components of $h_{a b c}$ are

$$
h_{012}=-h_{034}=-h_{512}=h_{534}=\text { const. }
$$

The derivation of M5-brane solitons in the constant $\mathcal{H}$ background is along the same line as the case with a zero $\mathcal{H}$ 
background $[12,13,27]$, except that one has to impose the boundary condition, Eq. (9) or (10), at the infinity of the worldvolume.

\section{SELF-DUAL STRING}

The self-dual string in the M5-brane worldvolume theory is interpreted as the boundary of an M2-brane ending on an M5-brane:

$$
\begin{array}{lllllll}
\text { M5: } & 1 & 2 & 3 & 4 & 5 & \\
\text { M2: } & 1 & & & & 6 .
\end{array}
$$

All fields of the self-dual string soliton are independent of the worldvolume coordinates $\left(x^{0}, x^{1}\right)$ of the string soliton. We denote the four M5-brane worldvolume indices for the directions transverse to the string soliton with a tilde, i.e., $\tilde{a}, \tilde{m}=2, \ldots, 5$. We let only one of the scalar fields, which we choose as $X^{1^{\prime}} \equiv \phi$, to be active. The bosonic worldvolume field ansatz for the string soliton is

$$
X^{1^{\prime}}=\phi, \quad h_{01 \tilde{a}}=v_{\tilde{a}}, \quad h_{\tilde{a} \tilde{b} \tilde{c}}=\epsilon_{\tilde{a} \tilde{b} \tilde{c} \tilde{d}} v^{\tilde{d}}
$$

with the remaining components of $h_{a b c}$ vanishing, along with the boundary condition $h_{01 \tilde{a}}=h \delta_{\tilde{a}}^{2}$ at infinity.

Substituting the field ansatz, Eq. (12), into Eq. (8), one obtains the following supersymmetry variation of fermions $\Theta$ :

$$
\begin{aligned}
\hat{\delta} \Theta= & -\frac{1}{2} \epsilon\left[\operatorname{det}\left(e^{-1}\right) \partial_{m} \phi \gamma^{m} \gamma_{1^{\prime}}\right. \\
& \left.-2 \gamma^{01}\left\{v^{\tilde{m}} \gamma_{\tilde{m}}+\operatorname{det}\left(e^{-1}\right) v_{\tilde{m}} \gamma^{\tilde{m}}\right\}\right] .
\end{aligned}
$$

If one requires spinors to satisfy the constraint

$$
\epsilon=\epsilon \gamma^{01} \gamma_{1^{\prime}},
$$

then from the Killing spinor equation $\hat{\delta} \Theta=0$ one obtains the following Bogomol'nyi condition on the fields:

$$
v_{\tilde{a}}=\frac{1}{2} \frac{\delta_{\tilde{a}}^{\tilde{m}} \partial_{\tilde{m}} \phi}{1+\sqrt{1+\left(\partial_{\tilde{m}} \phi\right)^{2}}},
$$

where $\left(\partial_{\tilde{m}} \phi\right)^{2} \equiv \delta^{\tilde{m} \tilde{n}} \partial_{\tilde{m}} \phi \partial_{\tilde{n}} \phi$.

Making use of Eq. (2) along with the field ansatz, Eq. (12), one obtains the following nonvanishing components of $\mathcal{H}$ :

$$
\begin{aligned}
& \mathcal{H}_{01 \tilde{m}}=\frac{1}{1+4 v^{2}} e_{\tilde{m}}^{\tilde{a}} v_{\tilde{a}}, \\
& \mathcal{H}_{\tilde{m} \tilde{n} \tilde{p}}=\frac{\sqrt{1+\left(\partial_{\tilde{m}} \phi\right)^{2}}}{1-4 v^{2}} \epsilon_{\tilde{m} \tilde{n} \tilde{p} \tilde{q}} e_{\tilde{a}}^{\tilde{q}} v^{\tilde{a}},
\end{aligned}
$$

where $v^{2} \equiv \delta^{\tilde{a} \tilde{b}} v_{\tilde{a}} v_{\tilde{b}}$. The vielbein $e_{m}^{a}$ associated with the induced metric $g_{m n}$ on the M5-brane worldvolume is given by $\left(e_{m}^{a}\right)=\operatorname{diag}\left(1,1, e_{\tilde{m}}^{\tilde{a}}\right)$, with $e_{\tilde{m}}^{\tilde{a}}=\delta_{\tilde{m}}^{\tilde{a}}+c \partial_{\tilde{m}} \partial^{\tilde{a}} \phi$, and where $c \equiv\left(-1+\sqrt{1+\delta^{\tilde{m} \tilde{n}} \partial_{\tilde{m}} \phi \partial_{\tilde{n}} \phi}\right) / \delta^{\tilde{m} \tilde{n}} \partial_{\tilde{m}} \phi \partial_{\tilde{n}} \phi$. These nonzero components of $\mathcal{H}$ simplify to the following forms after the Bogomol'nyi condition, Eq. (15), is substituted:

$$
\mathcal{H}_{01 \tilde{m}}=\frac{1}{4} \partial_{\tilde{m}} \phi
$$

$$
\mathcal{H}_{\tilde{m} \tilde{n} \tilde{p}}=\frac{1}{4} \epsilon_{\tilde{m} \tilde{n} \tilde{p} \tilde{q}} \delta^{\tilde{q} \tilde{r}} \partial_{r} \phi
$$

where $\epsilon_{\tilde{m} \tilde{n} \tilde{p} \tilde{q}}^{\tilde{q}}=e_{\tilde{m}}^{\tilde{a}} e_{\tilde{n}}^{\tilde{b}} e_{\tilde{p}}^{\tilde{c}} e_{\tilde{q}}^{\tilde{d}} \epsilon_{\tilde{a} \tilde{b} \tilde{c} \tilde{d}}$.

To find the expression for the scalar $\phi$, one has to solve the equation (5) for the scalar $X^{5^{\prime}}=\phi$. It has been shown [29] that generally the equation of motion $G^{m n} \nabla_{m} \nabla_{n} X^{a^{\prime}}$ $=0$ for the scalar $X^{a^{\prime}}$ implies $G^{m n} \partial_{m} \partial_{n} X^{a^{\prime}}=0$. Note, the worldvolume fields for self-dual string solutions are independent of the worldvolume coordinates $x^{0}$ and $x^{1}$, and it can be shown by applying Eq. (15) so that $G^{\tilde{m} \tilde{n}} \propto \delta^{\tilde{m} \tilde{n}}$. So, the scalar $\phi$ satisfies the flat Laplace's equation

$$
\delta^{\tilde{m} \tilde{n}} \partial_{\tilde{m}} \partial_{\tilde{n}} \phi=0
$$

From Eq. (17), one can see that the solution to Eq. (18), satisfying the boundary condition $\mathcal{H}_{012}=\left(h /\left(1+4 h^{2}\right)\right)$ $=\frac{1}{4} \sin \theta$ at infinity, describes an array of strings with charge $Q_{K}$ located at $x^{\tilde{m}}=y_{K}^{\tilde{m}}$ and given by

$$
\phi=\phi_{0}+\sum_{K} \frac{2 Q_{K}}{\left|x-y_{K}\right|^{2}}+x^{2} \sin \theta
$$

Due to the self-duality condition on $\mathcal{H}$, the string at $x^{\tilde{m}}$ $=y_{K}^{\tilde{m}}$ carries the same electric and magnetic charges, $Q_{E}$ $=Q_{M}=Q_{K}$. From the second equation of Eq. (16) or (17), one can see that the boundary condition $\mathcal{H}_{345}=-(h /(1$ $\left.\left.-4 h^{2}\right)\right)=-\frac{1}{4} \tan \theta$ at infinity is automatically satisfied by Eq. (19). From the expression (19) for the scalar $\phi$, one can see that the M2-brane is tilted towards the $x^{2}$ direction due to the force felt by the self-dual string at the boundary of the suspended M2-brane in the background of the constant $\mathcal{H}$ field. This force due to the constant $\mathcal{H}$ field is canceled by the tension of the M2-brane.

By compactifying the above self-dual string solution along the $x^{1}$ direction, one obtains the following 0-brane soliton (bion) on the D4-brane worldvolume in the constant $B$ field background studies of Refs. [16,17]:

$$
\mathcal{F}_{0 \tilde{m}}=\frac{1}{4} \partial_{\tilde{m}} \phi, \quad \phi=\phi_{0}+\sum_{K} \frac{2 Q_{K}}{\left|x-y_{K}\right|^{2}}+x^{2} \sin \theta,
$$

where $\mathcal{F}_{\tilde{m} \tilde{n}} \equiv \mathcal{H}_{\tilde{m} \tilde{n} 1}$. Dimensional reduction along, say, the $x^{5}$ direction leads to the following string soliton on the D4brane worldvolume: 


$$
\mathcal{F}_{\tilde{m} \tilde{n}}=\frac{1}{4} \epsilon_{\tilde{m} \tilde{n} \tilde{p}} \delta^{\tilde{p} \tilde{q}} \partial \tilde{q} \phi, \quad \phi=\phi_{0}+\sum_{K} \frac{2 Q_{K}}{\left|x-y_{K}\right|^{2}}+x^{2} \sin \theta,
$$

where $\mathcal{F}_{\tilde{m} \tilde{n}}=\mathcal{H}_{\tilde{m} \tilde{n} 5}$.

It is straightforward to show that a self-dual string on a calibrated surface in the constant $h_{a b c}$ field background also preserves supersymmetry. The amount of supersymmetry preserved depends on the type of calibrated surface. We denote $X^{I}$ as the scalars associated with the calibrated surface and, as above, $X^{1^{\prime}}=\phi$ is the scalar of the self-dual string soliton. The supersymmetry variation of the fermion is given by

$$
\begin{aligned}
\hat{\delta} \Theta= & -\frac{1}{2} \epsilon\left[\left\{\operatorname{det}\left(e^{-1}\right) \partial_{m} X^{I} \gamma^{m} \gamma_{I}\right.\right. \\
& -\frac{1}{3 !} \operatorname{det}\left(e^{-1}\right) \partial_{m_{1}} X^{I_{1}} \partial_{m_{2}} X^{I_{2}} \partial_{m_{3}} X^{I_{3}} \gamma^{m_{1} m_{2} m_{3}} \gamma_{I_{1} I_{2} I_{3}} \\
& \left.+\frac{1}{5 !} \operatorname{det}\left(e^{-1}\right) \partial_{m_{1}} X^{I_{1}} \ldots \partial_{m_{5}} X^{I_{5}} \gamma^{m_{1} \ldots m_{5}} \gamma_{I_{1} \ldots I_{5}}\right\} \\
& +\operatorname{det}\left(e^{-1}\right) \partial_{m} \phi \gamma^{m} \gamma_{1^{\prime}}-2 \gamma^{01} \\
& \left.\times\left(v^{\tilde{m}} \gamma_{\tilde{m}}+\operatorname{det}\left(e^{-1}\right) v_{\tilde{m}} \gamma^{\tilde{m}}\right)\right] .
\end{aligned}
$$

The self-dual string soliton on the calibrated surface preserves supersymmetry if it satisfies the Killing spinor equation $\hat{\delta} \Theta=0$ with nonzero $\epsilon$. We would rather consider two equations obtained by setting two separate terms, i.e., the terms in the curly bracket of Eq. (22) and the remaining terms, equal to zero. The first equation (associated with the terms in the curly bracket) determines the amount of supersymmetry preserved by and the geometry of the calibrated surface. The second equation is for the self-dual string soliton on the calibrated surface. The amount of preserved supersymmetry can be determined by considering the supersymmetry projectors of the associated intersecting M5-brane configuration and the added M2-brane. One can also add an M2-brane to an intersecting M5-brane configuration without breaking additional supersymmetry if the supersymmetry projectors associated with the M5-branes yield the supersymmetry projector for the added M2-brane. The scalar $\phi$ no longer satisfies the flat Laplace's equation, but satisfies an equation determined by the curved metric of the calibrated surface.

\section{NEUTRAL STRING}

We consider the worldvolume soliton counterpart to the following target space configuration where the $\mathrm{M}$ wave travels along a longitudinal direction of an M5-brane:

M5: $1 \begin{array}{lllll}1 & 2 & 3 & 4 & 5\end{array}$

MW: $\quad 5$.
When the $x^{5}$ direction is compactified, this configuration becomes a D0-brane in a D4-brane, where the D0-brane is interpreted as an instanton of the D4-brane worldvolume theory [30,31]. The worldvolume soliton counterpart to Eq. (23) has no active scalar and nonvanishing $\mathcal{H}_{0 \tilde{m} \tilde{n}}$ and $\mathcal{H}_{5 \tilde{m} \tilde{n}}$, where $\tilde{m}, \tilde{n}=1,2,3,4$. The nonzero component $\mathcal{H}_{5 \tilde{m} \tilde{n}}=F_{\tilde{m} \tilde{n}}$ is (anti-) self-dual as a two-form in four-dimensional Euclidean space, and gives rise to a string-like soliton in the $x^{5}$ direction. But such a string-like soliton does not carry a charge of the $\mathcal{H}$ field. So, such a soliton is called an instanton or a neutral string. The corresponding bosonic worldvolume field ansatz is

$$
h_{0 \tilde{a} \tilde{b}}= \pm h_{5 \tilde{a} \tilde{b}} \equiv F_{\tilde{a} \tilde{b}},
$$

where $\tilde{a}, \widetilde{b}=1, \ldots, 4$, and all of the scalar fields $X^{a^{\prime}}$ are set to zero (hence, the induced metric is flat, $g_{m n}=\eta_{m n}$ ). To consider an instanton string solution on the noncommutative M5-brane worldvolume, one has to impose the boundary condition such that $h_{m n p}$ is a nonzero constant at infinity. In order for the boundary condition to be compatible with the field ansatz, Eq. (24), one has to go to the infinite momentum frame (through infinite boost along the $x^{5}$ direction), in which the nonzero components of $h_{m n p}$ at infinity are given by Eq. (10). This boundary condition can be imposed on the field ansatz (24) only for the negative sign choice in Eq. (24), i.e., only when $F_{\tilde{m} \tilde{n}}$ is antiself-dual. ${ }^{1}$ Then, the supersymmetry variation Eq. (8), of the fermions takes the following form:

$$
\hat{\delta} \Theta=-\frac{1}{2} \epsilon F^{\tilde{m} \tilde{n}}\left(\gamma_{0 \tilde{m} \tilde{n}}-\gamma_{5} \tilde{m} \tilde{n}\right) .
$$

The resulting Killing spinor equation $\hat{\delta} \Theta=0$ can be satisfied if we impose the following constraint on spinors:

$$
\epsilon \gamma_{0} \gamma_{5}=\epsilon
$$

We show that a neutral string on a calibrated surface in the constant $h_{a b c}$ field background also preserves supersymmetry. For this purpose, it is convenient to introduce the light-cone coordinates

$$
u=\frac{1}{\sqrt{2}}\left(x^{0}-x^{5}\right), \quad v=\frac{1}{\sqrt{2}}\left(x^{0}+x^{5}\right) .
$$

In terms of the light-cone coordinates, the boundary condition, Eq. (10), on the $h_{a b c}$ field is

$$
h_{u 12}=-h_{u 34}=\text { const, }
$$

and the bosonic field ansatz (24) with the negative sign becomes

\footnotetext{
${ }^{1}$ The infinite boost along the negative $x^{5}$ direction would lead to the boundary condition on $h_{a b c}$ with opposite signs, and therefore select the self-dual $F_{\tilde{m} \tilde{n}}$ [i.e., the positive sign in Eq. (24)].
} 


$$
h_{u \tilde{a} \tilde{b}}:=\bar{F} \tilde{a} \tilde{b} .
$$

From Eq. (2), one can see that the only nonzero component of the corresponding $\mathcal{H}$ field is $\mathcal{H}_{u \tilde{m} \tilde{n}}=\bar{F} \tilde{m} \tilde{n}$. The supersymmetry variation of the fermion is

$$
\widetilde{\delta} \Theta=-\frac{1}{2} \epsilon\left[\bar{F}^{\tilde{m} \tilde{n}} \gamma_{v \tilde{m} \tilde{n}}+\bar{F}^{\tilde{m} \tilde{n}} \partial_{\tilde{m}} X^{I_{1}} \partial_{n} X^{I_{2}} \gamma_{v} \gamma_{I_{1} I_{2}}\right]
$$

where $X^{I}$ are scalars associated with the calibrated surface and $\epsilon$ is the spinor for supersymmetry preserved by the calibrated surface, determined by setting the terms in the curly bracket of Eq. (22) equal to zero. By imposing the spinor constraint $\epsilon \gamma^{u}=0$, one can set this supersymmetry variation to zero, making use of the relation $\gamma_{v}=\gamma^{u}$. The possibility of having such a supersymmetric configuration depends on whether the supersymmetry projectors of the intersecting M5-brane configuration associated with the calibrated surface yields the projector $\epsilon \gamma^{u}=0$ (or $\epsilon \gamma^{0} \gamma^{5}=\epsilon$ ). Had one used the boundary condition on $h_{a b c}$ corresponding to the infinitely boosted frame along the negative $x^{5}$ direction, one would have had a self-dual instanton string on a calibrated surface with both $\mathcal{H}_{v \tilde{m} \tilde{n}}:=\bar{G}_{\tilde{m} \tilde{n}}$ and the associated spinor constraint $\epsilon \gamma^{v}=0$.

\section{TRAVELING WAVE ON A CALIBRATED SURFACE}

In this section we show that a supersymmetric traveling wave on a calibrated surface exists even in the nonzero constant $h_{a b c}$ field background. A traveling wave on the M5brane worldvolume is regarded as a fluctuation in the shape of the calibrated surface. We consider the wave traveling in the $x^{5}$ direction. For the purpose of studying the traveling wave, it is convenient to work with the light-cone coordinates, Eq. (27), and constant background $h_{a b c}$ field of the form, Eq. (28), in the infinite momentum frame. We expect that either purely left-moving or right-moving waves moving at the speed of light, i.e., the case when scalar fields $X^{a^{\prime}}$ do not depend on either $u$ or $v$, preserves supersymmetry. We consider the case when $X^{a^{\prime}}$ is independent of $v$, i.e., $\partial_{v} X^{a^{\prime}}$ $=0$. Then, the supersymmetry transformation, Eq. (8), reduces to the following form:

$$
\begin{aligned}
\hat{\delta} \Theta= & -\frac{1}{2} \epsilon\left[\operatorname{det}\left(e^{-1}\right) \partial_{u} X^{a^{\prime}} \gamma^{u} \gamma_{a^{\prime}}\right. \\
& -\frac{1}{3 !} \operatorname{det}\left(e^{-1}\right) \partial_{u} X^{a_{1}^{\prime}} \partial_{m_{2}} X^{a_{2}^{\prime}} \partial_{m_{3}} X^{a_{3}^{\prime}} \gamma^{u m_{2} m_{3}} \gamma_{a_{1}^{\prime} a_{2}^{\prime} a_{3}^{\prime}} \\
& +\frac{1}{5 !} \operatorname{det}\left(e^{-1}\right) \partial_{u} X^{a_{1}^{\prime}} \partial_{m_{2}} X^{a_{2}^{\prime}} \ldots \partial_{m_{5}} X^{a_{5}^{\prime}} \gamma^{u m_{2}} \ldots m_{5} \\
& \times \gamma_{a_{1}^{\prime}} \ldots a_{5}^{\prime}-h^{v m_{2} m_{3}} \partial_{m_{2}} X^{a_{2}^{\prime}} \partial_{m_{3}} X^{a_{3}^{\prime}} \gamma_{v} \gamma_{a_{2}^{\prime} a_{3}^{\prime}} \\
& \left.-\frac{1}{3} h^{v m_{2} m_{3}} \gamma_{v m_{2} m_{3}}\right] .
\end{aligned}
$$

This supersymmetry variation can be set to zero by imposing the spinor constraint $\epsilon \gamma^{u}=0$. So, a traveling wave in the constant $h_{a b c}$ field background (28) with arbitrary dependence of $X^{a^{\prime}}$ on $u$ preserves supersymmetry. The analysis of the case with no dependence on $u$, i.e., $\partial_{u} X^{a^{\prime}}=0$, is along the same line. The corresponding supersymmetry projector is $\epsilon \gamma^{v}=0$ (or $\epsilon \gamma^{0} \gamma^{5}=-\epsilon$ ).

Note added. While this work was being completed, there appeared a paper [32] which has overlapping results with Sec. III of our paper. However, that paper [32] has a slightly different derivation of the self-dual string soliton than ours does.
[1] A. Connes, M. R. Douglas, and A. Schwarz, J. High Energy Phys. 02, 003 (1998).

[2] M. R. Douglas and C. Hull, J. High Energy Phys. 02, 008 (1998).

[3] N. Seiberg and E. Witten, J. High Energy Phys. 09, 032 (1999).

[4] N. Seiberg, L. Susskind, and N. Toumbas, J. High Energy Phys. 06, 021 (2000).

[5] J. L. Barbon and E. Rabinovici, Phys. Lett. B 486, 202 (2000).

[6] R. Gopakumar, S. Minwalla, N. Seiberg, and A. Strominger, J. High Energy Phys. 08, 008 (2000).

[7] E. Bergshoeff, D. S. Berman, J. P. van der Schaar, and P. Sundell, hep-th/0006112.

[8] G. Papadopoulos and P. K. Townsend, Phys. Lett. B 380, 273 (1996).

[9] A. Strominger, Phys. Lett. B 383, 44 (1996).

[10] P. K. Townsend, Phys. Lett. B 373, 68 (1996).

[11] P. K. Townsend, Nucl. Phys. B (Proc. Suppl.) 58, 163 (1997).

[12] P. S. Howe, N. D. Lambert, and P. C. West, Nucl. Phys. B515, 203 (1998)
[13] P. S. Howe, N. D. Lambert, and P. C. West, Phys. Lett. B 419, 79 (1998)

[14] C. G. Callan and J. M. Maldacena, Nucl. Phys. B513, 198 (1998).

[15] G. W. Gibbons, Nucl. Phys. B514, 603 (1998).

[16] K. Hashimoto, J. High Energy Phys. 07, 016 (1999).

[17] A. Hashimoto and K. Hashimoto, J. High Energy Phys. 11, 005 (1999).

[18] K. Hashimoto, H. Hata, and S. Moriyama, J. High Energy Phys. 12, 021 (1999).

[19] D. Mateos, Nucl. Phys. B577, 139 (2000).

[20] K. Hashimoto and T. Hirayama, hep-th/0002090.

[21] S. Moriyama, Phys. Lett. B 485, 278 (2000).

[22] P. S. Howe and E. Sezgin, Phys. Lett. B 394, 62 (1997).

[23] P. S. Howe, E. Sezgin, and P. C. West, Phys. Lett. B 399, 49 (1997).

[24] P. S. Howe, E. Sezgin, and P. C. West, Phys. Lett. B 400, 255 (1997).

[25] G. W. Gibbons and G. Papadopoulos, Commun. Math. Phys. 202, 593 (1999). 
[26] J. P. Gauntlett, N. D. Lambert, and P. C. West, Commun. Math. Phys. 202, 571 (1999).

[27] J. P. Gauntlett, N. D. Lambert, and P. C. West, Adv. Theor. Math. Phys. 3, 91 (1999).

[28] E. Bergshoeff, D. S. Berman, J. P. van der Schaar, and P. Sundell, hep-th/0005026.
[29] O. Barwald, N. D. Lambert, and P. C. West, Phys. Lett. B 463, 33 (1999).

[30] M. R. Douglas, hep-th/9512077.

[31] E. Witten, Nucl. Phys. B460, 541 (1996).

[32] Y. Michishita, J. High Energy Phys. 09, 036 (2000). 